\title{
Improving the Efficiency of Distributive and Clinical Services in Hospital Pharmacy by
}

Darold T Barnum, Karen L Shields, Surrey M Walton, and Glen T Schumock

Darold T Barnum, PhD, MBA, is Professor, Department of Managerial Studies and Department of Information \& Decision Sciences, College of Business Administration, University of Illinois at Chicago, Chicago, IL, USA.

Karen L Shields, BSPharm, is Corporate Pharmacy Consultant, Sisters of St. Francis Health Services, Inc., Mishawaka, IN, USA.

Surrey M Walton, $\mathrm{PhD}$, is Associate Professor, Center for Pharmacoeconomic Research and Department of Pharmacy Administration, College of Pharmacy, University of Illinois at Chicago, Chicago, IL, USA.

Glen T Schumock, PharmD, MBA, FCCP, is Director, Center for Pharmacoeconomic Research, and Associate Professor, Department of Pharmacy Practice, College of Pharmacy, University of Illinois at Chicago, Chicago, IL, USA.

Corresponding author information: Darold T Barnum, 601 South Morgan Street, Department of Managerial Studies (M/C 243), University of Illinois at Chicago, Chicago, IL 60607-7123 USA. Voice 1-312-996-3073, Fax 1-312-996-3559, Email dbarnum@ uic.edu.

Submitted for consideration for publication in the special issue on Performance Measurement in the Health sector 


\begin{abstract}
Three problems impede the assessment of hospital pharmacy efficiency. First, although multiple efficiency indicators are utilized to measure a large variety of activities, it has not been possible to validly measure overall efficiency. Second, there have been no widely-used clinical activity indicators, so key outputs often have not been accounted for. Third, there has been no effective methodology for identifying when declines in efficiency are normal random variations and when they represent true decreases in performance. This paper presents a procedure that simultaneously addresses these three problems. It analyzes data from a group of U.S. hospital pharmacies that collect an inclusive set of clinical and distributional indicators. It employs Data Envelopment Analysis to develop comprehensive efficiency measures from the numerous outputs and inputs. It applies statistical Panel Data Analysis to estimate confidence intervals within which each pharmacy's true efficiency resides, and to develop control charts for signaling when a pharmacy's efficiency has declined by more than can be attributed to random variation. This integrated efficiency evaluation system is transferable to other hospital pharmacy systems, thereby offering decision makers a better way of measuring, controlling and improving hospital pharmacy efficiency.
\end{abstract}

Key words: Data Envelopment Analysis; Panel Data Analysis; Hospital pharmacy; Efficiency; Performance 


\section{Improving the Efficiency of Distributive and Clinical Services in Hospital Pharmacy}

\section{Introduction}

A key factor in every hospital's operation is the performance of its pharmaceutical services.

Pharmaceuticals are central to patient care, and the products themselves represent a significant portion of hospital expenditures, as do the pharmacy personal responsible for managing them. If the system is not effective, then the quality of patient care suffers. If it is not efficient, then it contributes to unwarranted increases in the large and growing cost of hospital care.

Hospital pharmacy managers are charged with maintaining effectiveness and efficiency. To control efficiency, they have developed numerous output/input indicators for monitoring the large variety of tasks performed by their staffs [1]. However, because so many different ratios are involved, it is difficult to identify the system's overall efficiency, or the relative efficiency of its major components.

A second problem in hospital pharmacy productivity assessment has been the relative absence of measures for clinical pharmacy functions as opposed to distribution tasks [1]. Distribution tasks are those that involve the drug product and include activities such as medication doses provided, and intravenous (IV) or chemotherapy orders filled. Clinical functions include activities such as medication management, drug evaluation and selection, and reviewing patient drug use. Pharmacist time spent in clinical activities often results in substantial savings to the hospital in drug costs as well as improvements in patient outcomes, but such benefits are not measured by most existing productivity monitoring systems. Of course, adding clinical indicators to the already-large number of distributional indicators amplifies the need for a comprehensive measure that validly synthesizes a pharmacy's overall performance, as well as summarizing the overall performances of its clinical and distributional components.

A third problem grows out of the rising frequency with which data are reported and available for management decision-making. Hospital pharmacies are increasingly using sophisticated computerized information systems that track both distribution and clinical activities. Clearly an advantage of such computerized information systems is that they can provide frequent, detailed productivity data, thereby 
making it possible for pharmacy managers to more rapidly make staffing or operational changes to improve efficiency. However, substantial random variation is more likely to be present in data covering short time periods, because such data lack the smoothing effect present when averages cover longer time periods. This makes it difficult for managers to estimate a pharmacy's true efficiency, and to separate normal random variations from true control issues.

The purpose of this paper is to present a methodology by which hospital pharmacy managers can simultaneously address these three problems. First, we describe an innovative set of clinical and distributional measures that recently were adopted by a United States hospital system. Clinical measures offer proxies for drug cost savings and improved patient outcomes that result from clinical inputs. Distributional indicators each measure a single, distinct type of pharmaceutical output.

Second, we use Data Envelopment Analysis (DEA) to develop comprehensive and economically sound efficiency measures computed from the numerous distributional and clinical outputs and inputs. It should be noted that DEA is becoming very popular for measuring healthcare efficiency. For example, the Journal of Medical Systems published two DEA papers in 2008 [2, 3] and already has three more forthcoming papers [4-6]. To date, however, the use of DEA to measure hospital pharmacy efficiency has been demonstrated in only one paper [7]. Herein, we use DEA to estimate the efficiency of a pharmacy as a whole, and to separately estimate the efficiency of its clinical and distribution components.

Third, we illustrate the use of statistical Panel Data Analysis (PDA) to develop confidence intervals for the performance of each hospital's pharmacy over time. We utilize these confidence intervals to estimate the range within which each pharmacy's true mean efficiency occurs, and to develop control charts to be used to identify scores from each pharmacy that are too low to be considered random.

These procedures should be easily transferable for use by other hospital pharmacy systems, using any inputs and outputs deemed appropriate. Thus, they provide hospital pharmacy managers with generalizable tools for measuring, controlling and improving efficiency. 


\section{Data and methods}

Our sample consisted of 13 periods of bi-weekly data for the first six months of 2008, from a group of 12 hospitals that are members of a United States hospital system. These 12 hospital pharmacies recently adopted the common set of clinical and distributional output measures that we used in the analysis.

Inputs

Table 1 shows the inputs included in each of the three DEA analyses conducted. Analysis 1 assessed distribution efficiency, Analysis 2 clinical efficiency, and Analysis 3 overall efficiency. Inputs were distribution labor hours for the first analysis, clinical labor hours for the second, and the total of distributional, clinical, and overhead labor hours for the third. Overhead hours were defined as nondistributive and non-clinical time, and included management activities, quality assurance tasks, and other routine activities that were not directly related to either drug products or patient care.

Only labor inputs were used in these analyses. Labor is the most important and controllable input that impacts hospital pharmacy efficiency. While the cost of drugs (another potential input) used in the production process in hospital pharmacy is a significant resource, this is primarily considered a passthough (or "throughput"). All other operating and capital costs are relatively insignificant, are directly related to labor hours, and certainly could not be substituted for labor. Furthermore, our primary purpose was to access personnel efficiency.

\section{Outputs}

Table 1 also shows each output included in the three analyses. Only the seven distribution outputs were used in Analysis 1 and only the three clinical outputs were used in Analysis 2. The three outputs used in Analysis 3 were 1) an aggregation of the distributional outputs, 2) an aggregation of the clinical savings outputs, and 3) the total number of clinical interventions. 


\section{Distributional outputs}

Distribution outputs included in this analysis are defined in Table 2. Each output is associated with the production of one unit of activity within a specific category of drug product. Each product has an assigned relative value (weighting factor) that is based on the estimated (standard) time (in minutes) to complete that task. For each hospital and each pay period, we had data on the number of times each task/product was performed. That quantity was multiplied by the weighting factor to get the total for each distribution output, which was then used in our analysis. As shown in Table 1, there were seven distributional outputs used in Analysis 1. Because the distributional outputs were weighted by the time that each should take, we were able to aggregate them into a single output for use in Analysis 3.

\section{Clinical outputs}

There were three clinical output included in Analysis 2. These were the number of clinical interventions, the estimated soft dollar savings from those interventions, and the estimated hard dollar savings from those interventions. The data for each of the clinical outputs were available for each hospital and each pay period, and were generated by a clinical pharmacy electronic documentation system/program used at the facilities (see below).

The documentation system contains a set of pre-specified clinical activities (interventions) that are performed by pharmacists. In total there are 91 interventions or activities included in the program used by these hospitals. Examples of these include consulting a physician on proper antibiotic selection, recommending to switch a drug from the intravenous to oral route of administration, or discontinuing a drug no longer needed. During their work day, pharmacists record each intervention performed, which then allows management analysis of clinical interventions by type and by pay period.

Many of the interventions that pharmacists perform are anticipated to save the hospital money. For example, if a pharmacist makes a recommendation to a physician to discontinue a drug that is no longer needed then the hospital saves the cost of that drug. While it is true that in a fee-for-service environment the "savings" that we describe would actually result in reduced revenue for the hospital and thus a net 


\section{DEA model}

We utilized the Charnes-Cooper-Rhodes (CCR) input-oriented DEA model [8], adapted to permit “superefficiency" scores [9]. That is, instead of truncating input-oriented efficiency scores at one $(0 \leq \theta \leq 1)$, the model allows the scores to assume the entire range of $0 \leq \theta<\infty$ where $\theta<1$ is inefficient and $\theta \geq 1$ is efficient. Efficient pharmacies with $\theta>1$ are sometimes called "superefficient," because they could increase inputs by $(\theta-1) \times 100$ percent with constant output and still remain efficient. 
We used the CCR model because the hospital pharmacies in our dataset showed constant returns to scale. Constant returns to scale also were reported in the earlier DEA study [7] as well as a non-DEA study of hospital pharmacy efficiency [10]. We allowed superefficiency scores to avoid the censored variables generated by conventional DEA programs. That is, conventional DEA models yield technical efficiency scores that ignore part of the efficiency variation, because an efficient pharmacy's score of 1 remains unchanged if it becomes more productive by increasing outputs or decreasing inputs.

Superefficiency scores are an observable proxy for the latent variable values underlying conventional efficiency scores, and therefore contain all of the information available. They remove the need to estimate the latent values using Tobit, sample-selected, or truncated regression [11], and allow the analyst to utilize all available empirical information to estimate efficiency.

The DEA model is shown in Linear Program 1 (equations $1.1-1.5$ ). For each observation $j=1, \ldots, J$ there are data on $n=1, \ldots, N$ inputs and on $m=1, \ldots, M$ outputs, where $x^{j}=\left(x_{j 1}, \ldots, x_{j N}\right) \in \mathbb{R}_{+}^{N}$ and $y^{j}=\left(y_{j 1}, \ldots, y_{j M}\right) \in \mathbb{R}_{+}^{M}$. The DEA score $\theta$ estimates the technical efficiency of the target $k$.

$$
\min _{\lambda} \theta
$$

$$
\begin{aligned}
& \text { subject to } \quad \sum_{j=1}^{J} x_{j n} \lambda_{j} \leq \theta x_{k n} \quad n=1, \ldots, N \\
& \sum_{j=1}^{J} y_{j m} \lambda_{j} \geq y_{k m} \quad m=1, \ldots, M \\
& \lambda_{k}=0 \\
& \lambda_{j} \geq 0 \quad j=1, \ldots J ; j \neq k
\end{aligned}
$$

There are 13 time periods and 12 pharmacies, for a total of 156 observations. We applied DEA to the entire set of 156 observations rather than performing a separate DEA for each cross-sectional period, thereby computing a common frontier for observations from all periods. This often is called an “intertemporal frontier," because observations are compared across time periods [12]. 
For each target pharmacy $k$, DEA was executed for the different sets of inputs and outputs identified in Table 1. The first set measures the technical efficiency with which the seven distributional tasks are performed. The second set measures the technical efficiency with which the three clinical activities are executed. The third set measures overall physical efficiency of a pharmacy, that is, the combination of pure technical efficiency, intra-output allocative efficiency and intra-input allocative efficiency [13, 14].

\section{Statistical panel data analysis}

Hsiao [15, p. 1] defines a panel dataset as "one that follows a given sample of individuals over time, and thus provides multiple observations on each individual in the sample." Because our dataset consists of a sample of 12 pharmacies $(j=1, \ldots, 12)$, each reporting 13 bi-weekly observations $(t=1, \ldots, 13)$, we were able to utilize statistical Panel Data Analysis (PDA). PDA provides proven and powerful statistical techniques that are unavailable to either cross-sectional or time-series data analysis [15-19]. Most important for the present case, PDA offers methodologies for testing DEA efficiency score residuals for Normality, identical distribution across pharmacies, and independence across pharmacies and over time. These conditions must either be present or adjusted for in order to estimate valid confidence intervals within which each pharmacy's true efficiency occurs with a given probability.

Therefore, our panel data statistical model was:

$$
w_{j} \theta_{j t}=\alpha_{j}+\beta_{j}(t-1)+u_{j t}
$$

$\theta_{j t}$ is the efficiency score of pharmacy $j$ in period $t$. The response variable is the product of $\theta_{j t}$ and the weight $w_{j}$, using Equation 3 (below) to compute the weight based on the standard error of estimate for data from pharmacy $j . \alpha_{j}$ is the individual effect of pharmacy $j, \beta_{j}$ is the mean change per period in the individual effect $\alpha_{j}$ of pharmacy $j$, and $u_{j t}$ is the random error in the response variable of pharmacy $j$ in period $t$. Many pharmacies showed linear trends in efficiency over time, so Equation 2 includes a factor $(t-1)$ that adjusts mean efficiency for the year involved. If there is no change in the efficiency scores over 
time for pharmacy $j$, or if the temporal trend is inconsistent, then $\beta_{j}$ will not be statistically significant and $\alpha_{j}$ will be an unbiased estimate of the mean weighted performance score. Finally, note that in Equation $3, \alpha_{j}^{*}$ and $\beta_{j}^{*}$ are estimated using $\theta_{j t}$ as the response variable.

$$
w_{j}=1 / \sqrt{\sum_{t=1}^{T}\left(\theta_{j t}-\alpha_{j}^{*}-\beta_{j}^{*}(t-1)\right)_{j t}^{2} / T}
$$

The preceding model (2) assumes that the random errors $u_{j t}$ in the efficiency scores are independent and identically distributed (i.i.d.), and Normally distributed. Before any attempt to construct control charts or confidence intervals can be validly performed, it is necessary to confirm that the preceding assumptions hold. If the conditions do not hold, appropriate Generalized Least Squares (GLS) models would be necessary to account for violations. That is, one must empirically test the data to determine whether the data generating process has produced DEA scores that are (or are not) i.i.d. and Normally distributed, and then use statistical models that have been justified by the empirical evidence. We do so as explained next, using a 0.05 level of significance.

To test for Normal distribution of the errors, we used the Shapiro-Wilk W test, the Shapiro-Francia W' test, and a joint skewness and kurtosis test [20]. To test whether the errors were identically distributed, we used the Breusch-Pagan/Cook-Weisberg [17] test for heteroskedasticity across the pharmacies. (Because the pharmacies' error distributions were heteroskedastic when $\theta$ was used as the response variable, we obtained homoskedasticity by weighting each pharmacy's DEA scores by its standard error [21], per equation 3.)

The errors are not independent if either serial or contemporaneous correlation is present. Serial correlation, often called autocorrelation, occurs among a given panel member's error terms when the error of one time period is correlated with the errors of other time periods. We tested for serial correlation using the Wooldridge test [16] for autocorrelation in panel data. Contemporaneous correlation, also called cross-sectional correlation and spatial correlation, occurs when the error terms across panel members are correlated. Our tests for contemporaneous correlation included Friedman's $R_{A V E}$ statistic 


\section{Results}

\section{Distribution system technical efficiency}

First, consider the efficiency with which each pharmacy produces its seven distributional outputs with the input of distributional employee hours. Linear Program 1 was used to compute the DEA scores $\theta_{j t}$, then Panel Data Analysis using Equations 2 was applied to the DEA scores in order to 1) assure that the scores met statistical requirements, 2) estimate confidence intervals for each pharmacy's true technical efficiency, and 3) develop control charts for each pharmacy's technical efficiency.

\section{Statistical diagnostic tests}

The Breusch-Pagan/Cook-Weisberg test for heteroskedasticity showed no statistically significant differences among the pharmacies $\left[\chi^{2}<0.005, \mathrm{P}\left(\chi^{2}(11) \geq 0.005\right)=1.0000\right]$. The Shapiro-Wilk W test did not reject Normality $[\mathrm{z}=1.234, \mathrm{P}(\mathrm{z}>1.234)=0.108]$, nor did the Shapiro-Francia $\mathrm{W}^{\prime}$ test $[z=1.240$, $\mathrm{P}(z>1.240)=0.108]$, nor did the skewness/kurtosis test for Normality $\left[\chi^{2}=3.14, \mathrm{P}\left(\chi^{2}(2)>3.14\right)=\right.$ 0.208]. There was no statistically significant first-order serial correlation based on the Wooldridge test 
for autocorrelation in panel data $[\mathrm{F}=3.042, \mathrm{P}(\mathrm{F}(1,11)>3.042=0.109]$. Test results for cross-sectional independence of residuals included Freedman's $R_{A V E}\left[R_{A V E}=15.121, \mathrm{P}\left(R_{A V E}>15.121\right)=0.177\right]$,

Frees' $R_{A V E}^{2}\left[R_{A V E}^{2}=\quad-0.033, \mathrm{P}\left(R_{A V E}^{2}>0.1984=0.10\right]\right.$, Pesaran's $C D$ cross-sectional dependence test $[C D=0.777, \mathrm{P}(\mathrm{CD}>|0.777|)=0.437]$, and the Breusch-Pagan LM test of independence $\left[\chi^{2}=82.987\right.$, $\left.\mathrm{P}\left(\chi^{2}(66)>82.987\right)=0.077\right]$. Therefore, the null hypotheses that the residuals are i.i.d. and Normally distributed cannot be rejected, so we employed the standard i.i.d. and Normality assumptions. The fixedeffect panel regression based on Equation 2 had an R-square of $0.983[\mathrm{~F}=336.30, \mathrm{P}(\mathrm{F}(23,132)>336.30)$ $<0.00005]$, and trend variables were statistically significant $[\mathrm{F}=12.84, \mathrm{P}(\mathrm{F}(12,132)>12.84)<0.00005]$.

\section{Confidence intervals}

Figure 1 provides the range within which true efficiency of each hospital pharmacy's distribution system would occur, with 0.95 confidence, over the thirteen bi-week period. In terms of trends, downtrends with statistical significance at the 0.05 level were present at Hospital Pharmacy 2, Hospital Pharmacy 4, Hospital Pharmacy 5, and Hospital Pharmacy 12, and a statistically significant uptrend was present at Hospital Pharmacy 8. Overall, the lowest efficiencies were found at Hospital Pharmacy 3 and Hospital Pharmacy 12. So, in terms of distribution system efficiency, the pharmacy that should be examined first would be Hospital Pharmacy 12 because of its low efficiency and downward trend.

\section{Control charts}

Figure 2 shows the control charts for each pharmacy. If the most recent observation is at or below the bottom line of the chart, then its low efficiency would occur by chance only 10 percent of the time. In this event, it is likely that its efficiency score is not just a random variation, but really is lower than expected. In such cases, an immediate examination is in order. Although it does not appear from the graph that any pharmacies were substantially outside their lower control limits in period 13, it would 
probably be worthwhile to examine Hospital Pharmacy 11 and possibly Hospital Pharmacy 3 because both already were reporting quite low efficiencies.

\begin{abstract}
Clinical system technical efficiency
Next, consider the technical efficiency with which each pharmacy produced its three clinical outputs, namely number of interventions, soft dollar savings and hard dollar savings, with the sole input being clinical work hours. Again, Linear Program 1 was used to compute the DEA scores $\theta_{j t}$.
\end{abstract}

\title{
Statistical diagnostic tests
}

The Breusch-Pagan/Cook-Weisberg test for heteroskedasticity found no statistically significant differences among the pharmacies $\left[\chi^{2}=0.00, \mathrm{P}\left(\chi^{2}(11)>0.005\right)=1.0000\right]$. The Shapiro-Wilk W test did not reject Normality $\left[z=-0.201 \mathrm{P}(\mathrm{z}>-0.201)=0.580\right.$, nor did the Shapiro-Francia $\mathrm{W}^{\prime}$ test $[z=0.216$, $\mathrm{P}(z>0.216)=0.415]$, nor did the joint skewness/kurtosis test for Normality $\left[\chi^{2}=0.88, \mathrm{P}\left(\chi^{2}(2)>0.88\right)\right.$ $=0.644]$. There was no statistically significant first-order serial correlation based on the Wooldridge test for autocorrelation in panel data $[\mathrm{F}=0.003, \mathrm{P}(\mathrm{F}(1,11)>0.003=0.961]$. Likewise, there was no statistically significant contemporaneous correlation as shown by Freedman's $R_{A V E}\left[R_{A V E}=12.077\right.$, $\left.\mathrm{P}\left(R_{A V E}>12.077\right)=0.358\right]$, Frees' $R_{A V E}^{2}\left[R_{A V E}^{2}=-0.166, \mathrm{P}\left(R_{A V E}^{2}>0.1984=0.10\right]\right.$, Pesaran's $C D$ crosssectional dependence test $[\mathrm{CD}=0.213, \mathrm{P}(\mathrm{CD}>|0.231|)=0.831]$, and the Breusch-Pagan $\mathrm{LM}$ test of independence $\left[\chi^{2}=84.630, \mathrm{P}\left(\chi^{2}(66)>84.630\right)=0.061\right]$. Therefore, the null hypotheses that the residuals are i.i.d. and Normally distributed cannot be rejected, so we employed the standard assumptions. The regression based on Equation 2 had an R-square of $0.985[\mathrm{~F}=381.22 \mathrm{P}(\mathrm{F}(23,132)>381.22)<$ $0.00005]$, and trends were statistically significant $[\mathrm{F}=1.86, \mathrm{P}(\mathrm{F}(12,132)>1.86)=0.045]$. 


\section{Confidence intervals}

Figure 3 provides the range within which true efficiency of each hospital pharmacy's clinical outputs would occur, with 0.95 confidence, over the thirteen bi-week period. The lowest estimated efficiencies occurred at Hospital Pharmacy 1, Hospital Pharmacy 2, Hospital Pharmacy 4 and Hospital Pharmacy 9 (which also had the greatest variation). In terms of trends, a downtrend with statistical significance at the 0.05 level was present at Hospital Pharmacy 2, and a statistically significant uptrend was present at Hospital Pharmacy 3. So, in terms of clinical system efficiency, the hospital that should be examined first is certainly Hospital Pharmacy 2 because of its low efficiency and a downward trend, and second might be Hospital Pharmacy 9 because of its generally low clinical efficiency and its large variation.

\section{Control charts}

Figure 4 offers the control charts for each pharmacy's clinical system. Hospital Pharmacy 2's latest efficiency score was on the lower limit, which may indicate that its already troublesome downtrend is getting steeper, so Hospital Pharmacy 2 warrants immediate and close examination. Although Hospital Pharmacy 3 was experiencing increasing clinical efficiency, its last observation was on its lower limit so it would be worthwhile to check it out to be sure that all is well.

\section{Total Hospital Pharmacy technical and physical allocation efficiency}

For a pharmacy as a whole, there were inputs to and outputs from the distribution system, inputs to and outputs from the clinical system, and "overhead" or fixed inputs necessary for the effective and efficient functioning of the pharmacy. Results in this section are for the overall efficiency of a hospital pharmacy. Recall that the single input was aggregated labor hours, which included distribution labor hours, clinical labor hours, and overhead/fixed labor hours. The outputs were aggregated distribution (sum of seven types of distribution outputs), aggregated clinical savings (sum of hard savings and soft savings), and clinical interventions (which cannot be aggregated with the other clinical outputs). 


\section{Technical vs. physical allocation efficiency}

Because inputs and outputs were aggregated to the greatest extent possible, this indicator included technical efficiency, intra-input allocation efficiency, and intra-output allocation efficiency [13, 14, 25, 26], while the preceding distributional and clinical efficiency indicators measured mainly technical efficiency. To explain the difference, consider the following. Suppose one hour of labor input can efficiently produce either 2 units of output A or 10 units of output B. Hospital Pharmacy 1 and Hospital Pharmacy 2 are both efficient, but Hospital Pharmacy 1 is required to produce 100 units A and 10 units of B while Hospital Pharmacy 2 is required to produce 10 units of A and 100 units of B. Because both pharmacies are technically efficient, Hospital Pharmacy 1 will use 50 hours of labor to produce its A and 1 hour to produce its B, for a total of 51 labor hours to produce 110 output units. Hospital Pharmacy 2 will use 5 hours of labor to produce its A and 10 hours of labor to produce its B, for a total of 15 hours to produce its 110 output units. If a DEA is run on these pharmacies, each with two labor inputs and two outputs then both will be found technically efficient. However, if the inputs and outputs are aggregated, then A's absolute efficiency is $110 / 51=2.2$ while B's absolute efficiency is $110 / 15=7.3$, so if B is assigned an efficiency score of 1 , then A's score will be 2.2/7.3 $=0.30$. The latter score incorporates both physical allocation and technical efficiency. Typically pharmacies do not have a choice on how much of each output must be produced, so, even if it is allocatively inefficient, each must produce what is required. However, from central management's perspective, it is worthwhile to know what the total efficiency of each pharmacy is, because in some cases outsourcing or other alternatives might be used to better allocate resources. Therefore, by aggregating inputs and outputs to the greatest extent possible, we measure total physical efficiency including both technical and physical allocation efficiency.

Although this indicator could be calculated by including only aggregated inputs from the clinical and distributional operations, a third input, overhead labor hours was also included. Thus, we have a comprehensive measure of the efficiency of each pharmacy that tells us the total physical efficiency with which a pharmacy operates. 


\section{Statistical diagnostic tests}

The Breusch-Pagan/Cook-Weisberg test for heteroskedasticity found no statistically significant differences among the pharmacies $\left[\chi^{2}=0.00, \mathrm{P}\left(\chi^{2}(11)>0.005\right)=1.0000\right]$. The Shapiro-Wilk W test did not reject Normality $\left[z=1.051 \mathrm{P}(\mathrm{z}>1.051)=0.147\right.$, nor did the Shapiro-Francia $\mathrm{W}^{\prime}$ test $[z=0.833$, $\mathrm{P}(z>0.833)=0.202]$, nor did the joint skewness/kurtosis test for Normality $\left[\chi^{2}=3.17, \mathrm{P}\left(\chi^{2}(2)>3.17\right)\right.$ $=0.205]$. There was no statistically significant first-order serial correlation based on the Wooldridge test for autocorrelation in panel data $[\mathrm{F}=0.028, \mathrm{P}(\mathrm{F}(1,11)>0.028=0.871]$. Likewise, there was no statistically significant contemporaneous correlation as shown by Freedman's $R_{A V E}\left[R_{A V E}=18.429\right.$, $\left.\mathrm{P}\left(R_{A V E}>18.429\right)=0.072\right]$, Frees' $R_{A V E}^{2}\left[R_{A V E}^{2}=-0.069, \mathrm{P}\left(R_{A V E}^{2}>0.1984=0.10\right]\right.$, and Pesaran's $C D$ cross-sectional dependence test $[\mathrm{CD}=1.562, \mathrm{P}(\mathrm{CD}>|1.562|)=0.118]$, although the Breusch-Pagan

LM test of independence was statistically significant $\left[\chi^{2}=93.72, \mathrm{P}\left(\chi^{2}(66)>93.572\right)=0.014\right]$. However, since three of the four tests indicated no contemporaneous correlation, we adopted the conservative decision and made no corrections for it (which results in slightly wider confidence intervals than would occur if contemporaneous correlation were adjusted for). Therefore, we employed the standard assumptions. The regression based on Equation 2 had an R-square of $0.969[\mathrm{~F}=178.22$ $\mathrm{P}(\mathrm{F}(23,132)>178.22)<0.00005]$, and the trends were statistically significant $[\mathrm{F}=2.28, \mathrm{P}(\mathrm{F}(12,132)>$ $2.28)=0.012]$. (Note: because there was only one input and three outputs, it was possible to perform a DEA separately on each cross-section of 13 pharmacies, and then to conduct diagnostics for i.i.d. and Normality on the panel of these cross-sectional efficiency scores. Decisions based on these test outcomes were the same as for the intertemporal DEA that we did use.)

\section{Confidence intervals}

Figure 5 provides the range within which true efficiency of each hospital pharmacy will occur, with 0.95 confidence, over the thirteen bi-week period. The lowest mean efficiencies occurred at Hospital 
Pharmacy 2 and Hospital Pharmacy 9. In terms of trends, a downtrend with statistical significance at the 0.05 level was present at Hospital Pharmacy 2 and Hospital Pharmacy 4, and a statistically significant uptrend was present at Hospital Pharmacy 3 and Hospital Pharmacy 8. So, in terms of overall system efficiency, the hospitals that should be examined first would be Hospital Pharmacy 2 and Hospital Pharmacy 4, because of their downward trends.

\section{Control charts}

Figure 6 offers the control charts for each pharmacy. In the present case Hospital Pharmacy 5's latest efficiency score was on the lower limit. And, again, although Hospital Pharmacy 3 was experiencing increasing efficiency, its last observation was on its lower limit so it would be worthwhile to check.

\section{Discussion}

In order to improve a hospital pharmacy's efficiency, it first is necessary to validly measure all key aspects of that efficiency. Developing a methodology to do so is the primary purpose of this paper.

After the efficiency indicators are collected, it next is necessary to identify the causes behind their values and determine what actions can be taken to improve them. Illustrating this post-collection analysis is beyond the scope of this already-long paper. But, detailed case analyses will be included in a companion paper currently being prepared. The companion paper focuses on the institutional pharmacy aspects of the procedures rather than methodology, and its audience is hospital pharmacy managers.

However, a brief description of the post-collection procedure may be of interest. General environmental factors or factors specific to a given hospital or pharmacy may influence the scores. Quantitative or qualitative methods can be used to adjust for such influences. In some cases, there may be uncorrectable factors or other justifiable reasons for low scores, or it may not be possible to take actions necessary to improve efficiency. In other cases, low scores may identify operations that management can and should improve. In all cases, the reported scores should be jointly examined by pharmacy management personnel and the quantitative analysts. The purpose of the procedure is to provide useful 
information about a specific hospital pharmacy. This can only occur with the joint involvement of managers familiar with the pharmacy being examined, and analysts skilled in the quantitative methods being applied.

On another subject, this paper demonstrates the use of Panel Data Analysis to develop valid probability estimates of the range within which an organization's true efficiency occurs, and the use of statistics to develop control charts. However, in developing confidence intervals it is necessary to consider the possibility of contemporaneous correlation, among other things. The main reason for contemporaneous correlation in DEA is that each organization's efficiency score is influenced by the performance of its efficient peer organizations. If certain efficient organizations systematically influence certain other organizations, it may cause correlation among their error terms. Therefore, often it is assumed that DEA score residuals will not be independent, so use of conventional parametric statistical models is inappropriate. One reason that we have provided empirical detail on the results of our statistical tests is to refute the assumption that i.i.d. and Normality never are present. The main reason for the statistical detail, is, of course, to illustrate the process.

However, although the results of the diagnostic tests may not always have to be presented in such detail as we do herein, they always must be conducted, because sometimes the conventional assumptions will be violated. If they are violated, then one can utilize Generalized Least Squares (GLS) models that correct for violations such as serial and/or contemporaneous correlation. When the number of panel members exceeds the number of time periods, one applicable GLS model is the Driscoll and Kraay [27] estimator. It yields a nonparametric variance-covariance matrix estimator with standard errors that are robust to serial and contemporaneous correlation [28]. When the number of time periods exceeds the number of panel members, then the Prais-Winsten estimator [17] is also available. It computes parametric variance-covariance estimates that are robust to contemporaneous and serial correlation.

Finally, because we had 12 hospitals but only 13 periods, we included all periods in our computations. However, in order to increase the power of the models to identify variations that are not random but a true change, the final period should be excluded from both the DEA and PDA models so its variation does not 
influence the results. So, were we to analyze the results in period 14, then the 13 -period control charts would be used, and analysis of results for period 15 would involve14-period control charts. For the present case, we believe that the maximum number of two-week periods that should be included in the models is 26 , which represents one year of data. Once that number is reached, we believe that the most recent 26 periods, excluding the period under review, would be preferable.

\section{Conclusions}

The efficient choice of pharmaceuticals and the efficiency of their delivery are key to the successful control of hospital costs. As a result, hospital pharmacy managers have developed a plethora of output/input ratios to measure the efficiency. However, such indicators fall far short of what is possible in the current environment, for several reasons.

First, because there are so many ratios, it is not possible to identify which actions will maximize overall efficiency, or how different pharmacies compare in their total performance.

Second, in spite of their large number, indicators have mainly been limited to tasks involving physical distribution of pharmaceuticals because these activities are easy to measure. Clinical activities, which have been considered difficult if not impossible to measure, actually may contribute more in total because they can result in savings on drug costs as well as enhanced patient outcomes. However, although the addition of clinical indicators is essential, their presence makes the problem of measuring overall pharmacy performance even more intractable.

Third, the recent growth in computerized pharmacy information systems makes possible the rapid availability of a great deal of detailed information. But, this new avalanche of data makes it even more difficult to know what to do to improve overall performance. Also, because the data covers very short time periods, there can be large random variations that do not reflect a system out of control. So, although the new technology makes quick and precise management action possible, its quantity and frequency can easily overwhelm decision makers. 

compatible and comprehensive distributional and clinical output measures, some of which are only available because of the computerized information system. As a result, all of the key distributional and clinical outputs are included. Second, it suggests DEA models that can separately summarize the efficiency of the pharmacies' clinical and distributional activities, with a third model that estimates the comprehensive efficiency of the pharmacy as a whole. Third, through the use of statistical PDA models, it suggests a methodology for developing statistical control charts, so pharmacy managers can quickly and accurately distinguish when conditions are indeed out of control and when the reports merely reflect random variations. We believe that the simultaneous use of these suggestions could provide significant assistance to hospital pharmacies seeking to increase and maintain their efficiency. 
Reference List

1. Gupta, S.R., Wojtynek, J.E., Walton, S.M., Botticelli, J.T., Shields, K.L., Quad, J.E., and Schumock, G.T., Pharmacy staffing and productivity. Am. J. Health. Syst. Pharm. 64(22): 23232324, 2006.

2. Nayar, P. and Ozcan, Y., Data Envelopment Analysis Comparison of Hospital Efficiency and Quality. J. Med. Syst. 32(3): 193-199, 2008.

3. Kirigia, J., Emrouznejad, A., Cassoma, B., Asbu, E., and Barry, S., A Performance Assessment Method for Hospitals: The Case of Municipal Hospitals in Angola. J. Med. Syst. 32(6): 509-519, 2008.

4. Polisena, J., Laporte, A., Coyte, P., and Croxford, R., Performance Evaluation in Home and Community Care. J. Med. Syst. 2009 (In Press), http://dx.doi.org/10.1007/s10916-008-9240-y.

5. Lee, K.H., Yang, S.B., and Choi, M., The Association between Hospital Ownership and Technical Efficiency in a Managed Care Environment. J. Med. Syst. 2009 (In Press), http://dx.doi.org/10.1007/s10916-008-9192-2.

6. Kontodimopoulos, N., Papathanasiou, N., Tountas, Y., and Niakas, D., Separating Managerial Inefficiency from Influences of the Operating Environment: An Application in Dialysis. J. Med. Syst. 2009 (In Press), http://dx.doi.org/10.1007/s10916-009-9252-2.

7. Schumock, G.T., Shields, K.L., Walton, S.M., and Barnum, D.T., Data envelopment analysis - A method for comparing hospital pharmacy productivity. Am. J. Health. Syst. Pharm. 2009 (In Press),

8. Charnes, A., Cooper, W.W., and Rhodes, E., Measuring the efficiency of decision making units. Eur. J. Oper. Res 2(6): 429-444, 1978.

9. Andersen, P. and Petersen, N.C., A procedure for ranking efficient units in data envelopment analysis. Manag. Sci. 39(10): 1261-1265, 1993.

10. Gupta, S.R., Wojtynek, J.E., Walton, S.M., Botticelli, J.T., Shields, K.L., Quad, J.E., and Schumock, G.T., Association between hospital size and pharmacy department productivity. Am. J. Health. Syst. Pharm. 64(9): 937-944, 2007.

11. Breen, R., Regression Models: Censored, Sample-Selected, or Truncated Data. Sage Publications, Thousand Oaks, CA, 1996.

12. Tulkens, H. and Vanden Eeckaut, P., Nonparametric efficiency, progress and regress measures for panel-data - methodological aspects. Eur. J. Oper. Res 80(3): 474-499, 1995.

13. Barnum, D.T. and Gleason, J.M., Biases in technical efficiency scores caused by intra-input aggregation: Mathematical analysis and a DEA application using simulated data. Appl. Econ. 38: 1593-1603, 2006.

14. Barnum, D.T. and Gleason, J.M., Technical efficiency bias in data envelopment analysis caused by intra-output aggregation. Appl. Econ. Lett. 14(9): 623-626, 2007. 
15. Hsiao, C., Analysis of Panel Data. Cambridge University Press, Cambridge, 2003.

16. Wooldridge, J.M., Econometric Analysis of Cross Section and Panel Data. MIT Press, Cambridge, MA, 2002.

17. Greene, W.H., Econometric Analysis, 6th Ed. Prentice Hall, Upper Saddle River, NJ, 2008.

18. Frees, E.W., Longitudinal and Panel Data: Analysis and Applications in the Social Sciences. Cambridge University Press, Cambridge, U.K, 2004.

19. Baltagi, B.H., Econometric Analysis of Panel Data. John Wiley, West Sussex, England, 2008.

20. StataCorp, Stata Base Reference Manual Release 10. StataCorp, College Station, Texas, 2007.

21. Carroll, R.J. and Ruppert, D., Transformation and Weighting in Regression. Chapman and Hall, New York, 1988.

22. Friedman, M., The use of ranks to avoid the assumption of normality implicit in the analysis of variance. J. Am. Stat. Assoc. 32: 675-701, 1937.

23. Frees, E.W., Assessing cross-sectional correlation in panel data. J. Econometrics 69(2): 393-414, 1995.

24. De Hoyos, R.E. and Sarafidis, V., Testing for cross-sectional dependence in panel-data models. Stata J. 6(4): 482-496, 2006.

25. Barnum, D.T. and Gleason, J.M., Measuring efficiency in allocating inputs among outputs with DEA. Appl. Econ. Lett. 13(6): 333-336, 2006.

26. Barnum, D.T. and Gleason, J.M., Technical efficiency bias caused by intra-input aggregation in data envelopment analysis. Appl. Econ. Lett. 12(13): 785-788, 2005.

27. Driscoll, J.C. and Kraay, A.C., Consistent covariance matrix estimation with spatially dependent panel data. Rev. Econ. Stat. 80(4): 549-560, 1998.

28. Hoechle, D., Robust standard errors for panel regressions with cross-sectional dependence. Stata J. 7(3): 281-312, 2007. 
Figure 1. True Mean Efficiencies of Pharmacy Distribution Systems, 95\% Confidence Interval
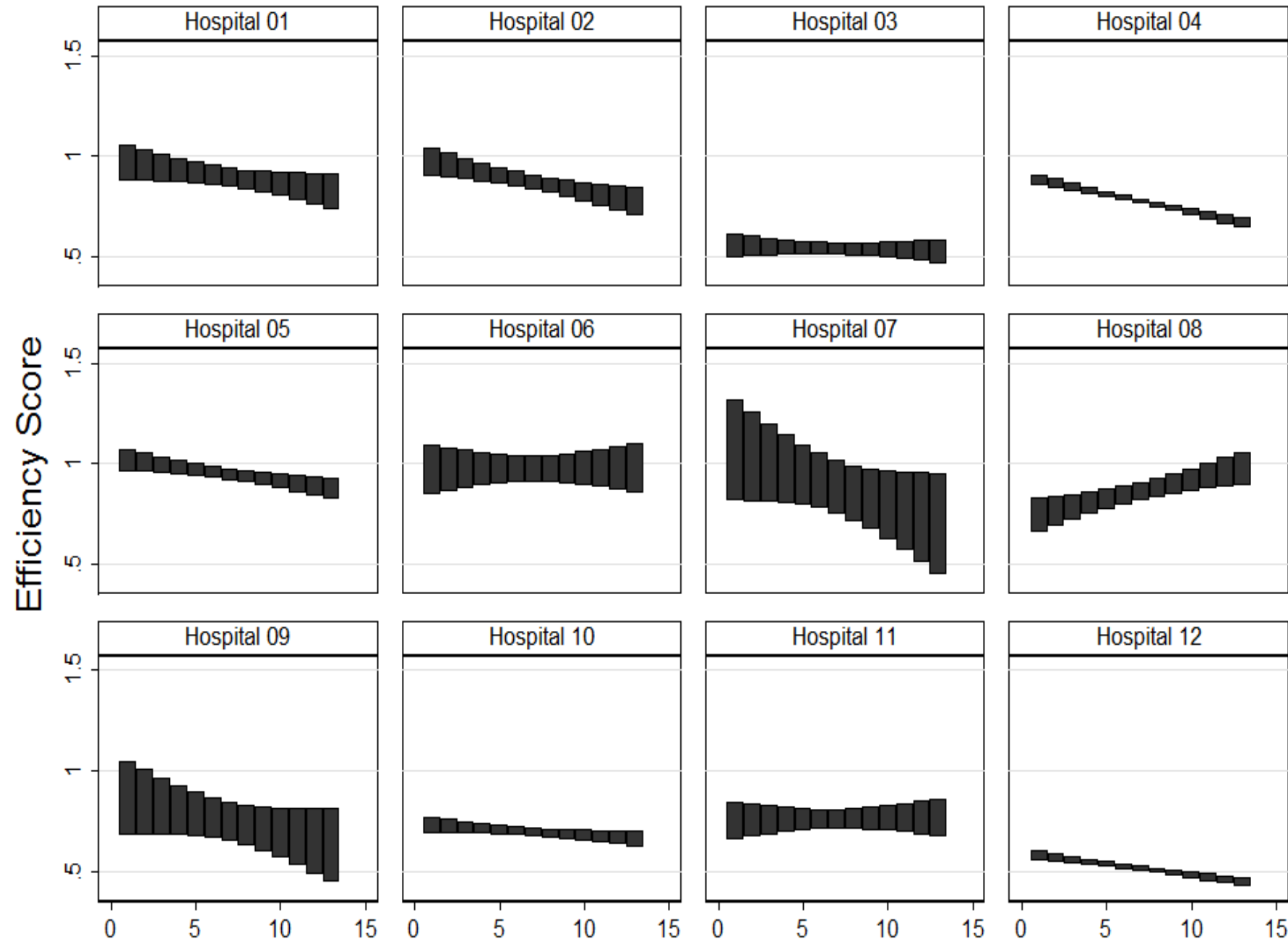

Graphs by Hospital Number

period 
Figure 2. Control Charts for Pharmacy Distribution Systems, and Actual Efficiency Scores

( $10 \%$ probability that actual efficiency will be below bottom line by random chance)
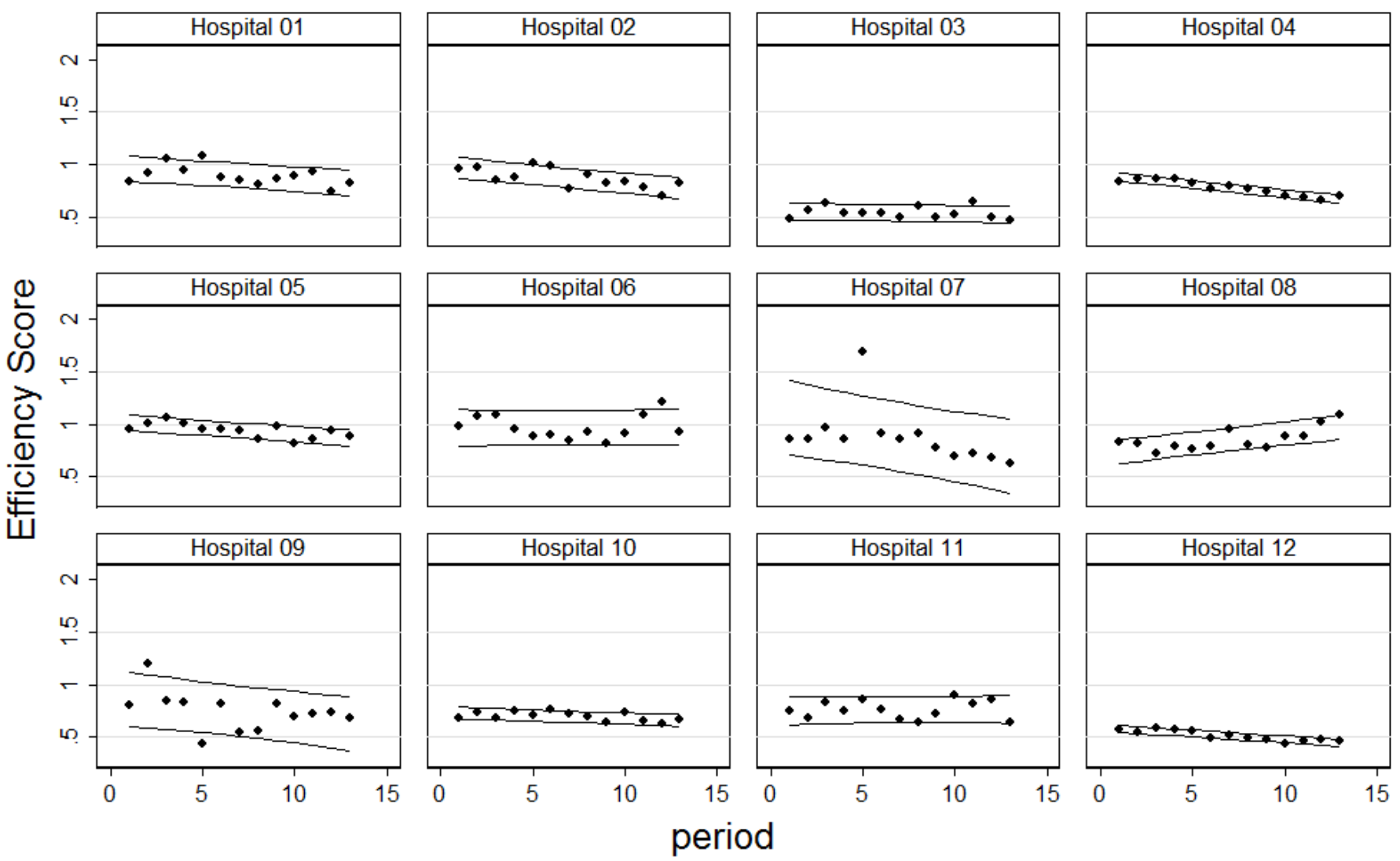
Figure 3. True Mean Efficiencies of Pharmacy Clinical Systems, 95\% Confidence Interval
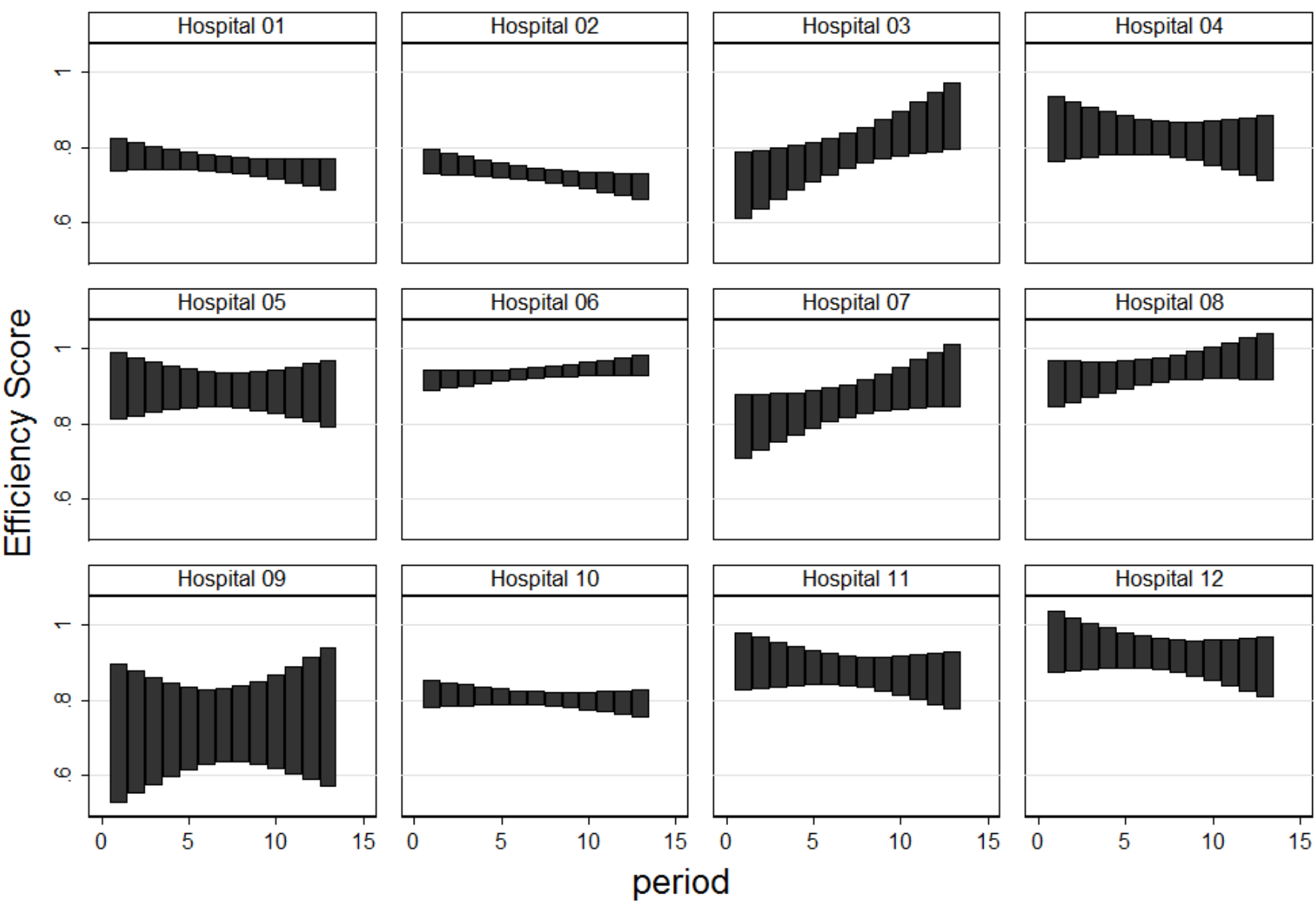

Graphs by Hospital Number 
Figure 4. Control Charts for Pharmacy Clinical Systems, and Actual Efficiency Scores

( $10 \%$ probability that actual efficiency will be below bottom line by random chance)
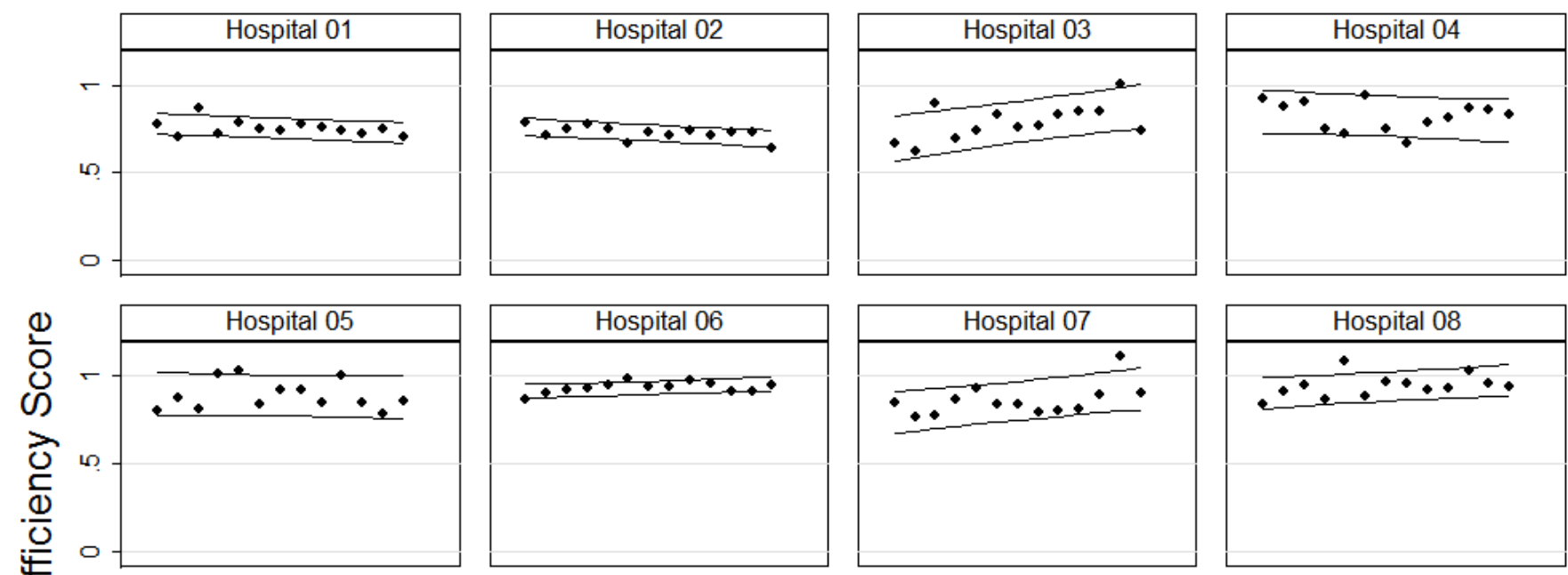

28
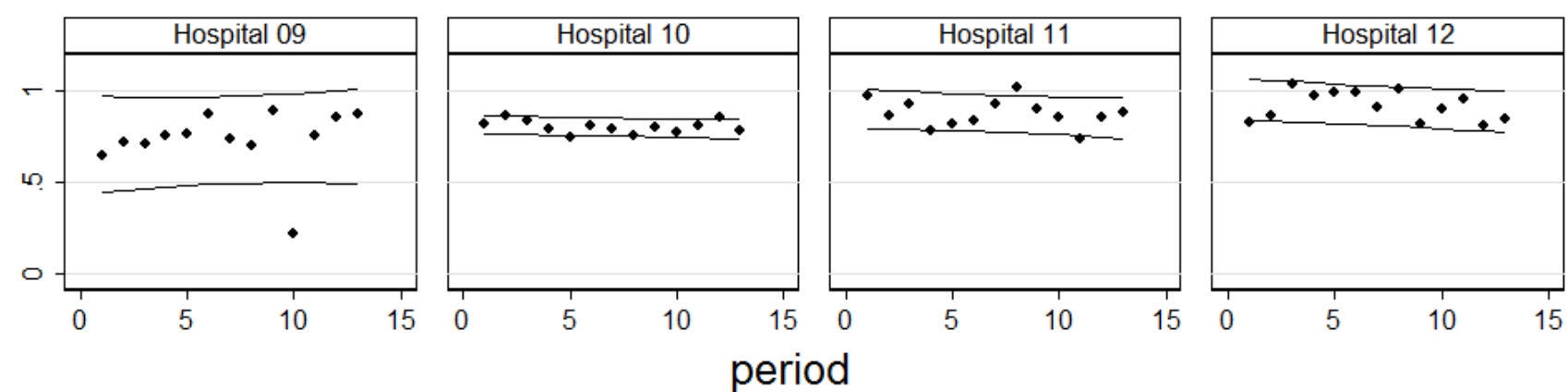

period

\section{- Actual Efficiency _ Upper Limit/Lower Limit}

Graphs by Hospital Number 

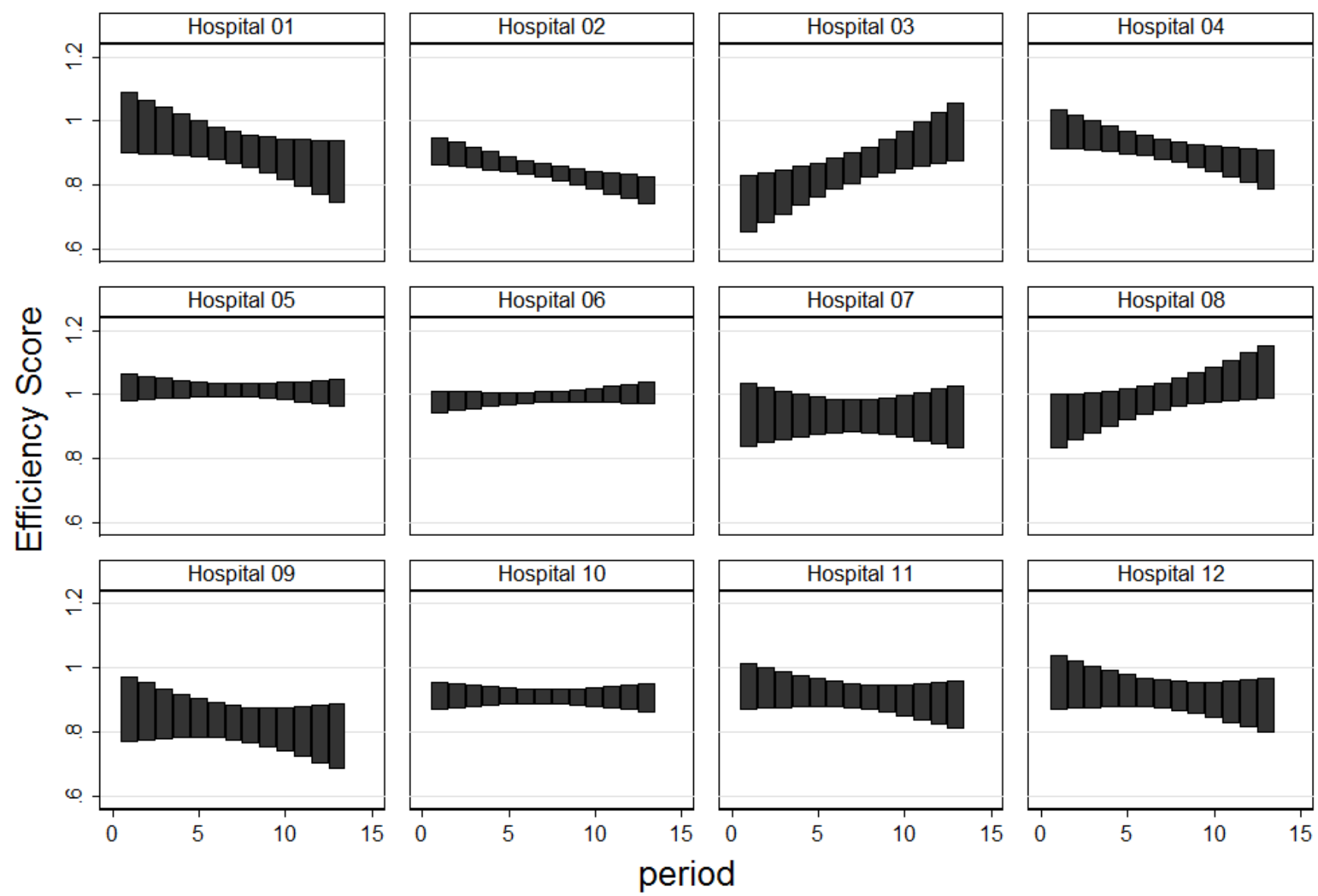

Graphs by Hospital Number

\section{period}

Graphs by Hospital Number 
Figure 6. Control Charts for Overall Pharmacy Efficiency, and Actual Efficiency Scores ( $10 \%$ probability that actual efficiency will be below bottom line by random chance)
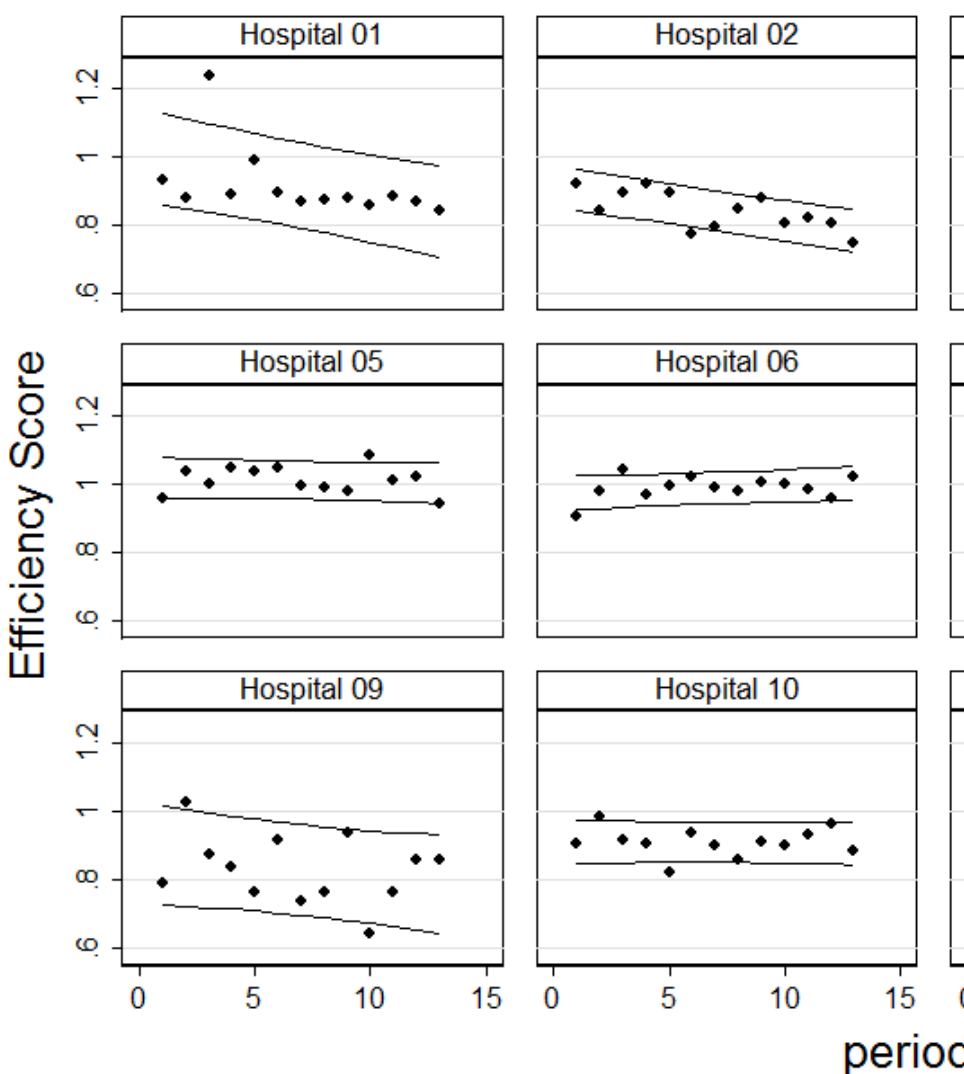
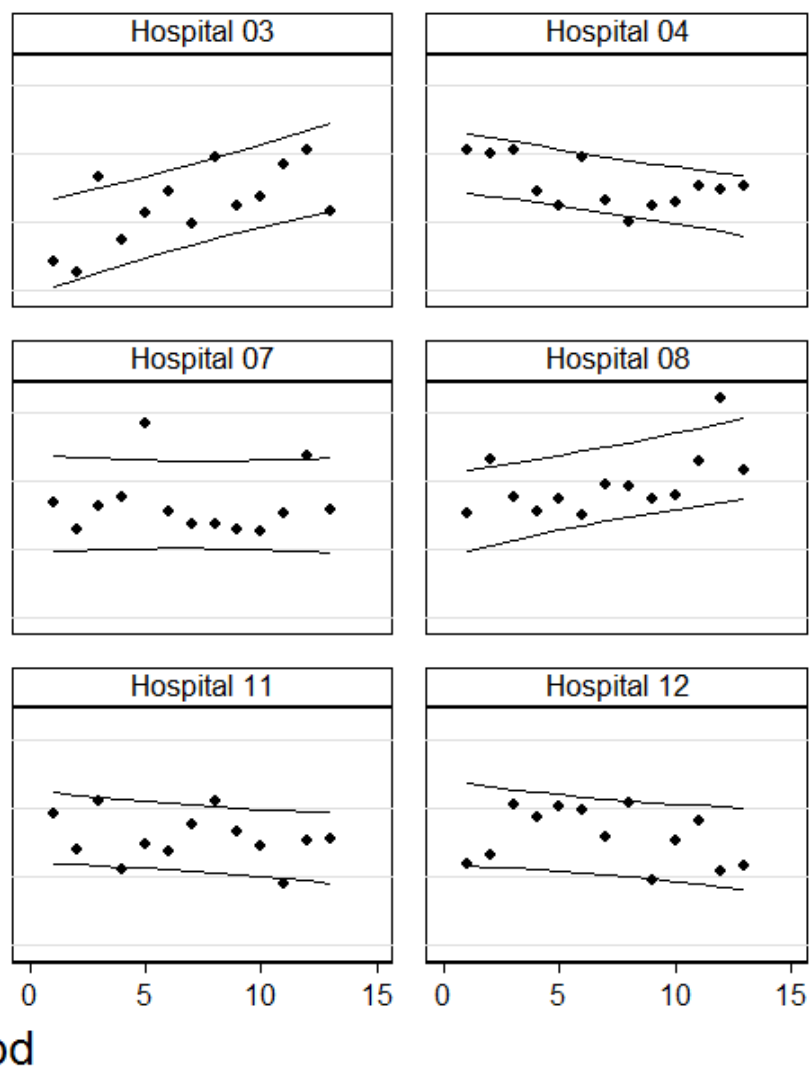

- Actual Efficiency

Graphs by Hospital Number 
Table 1. Inputs and outputs included in each DEA analysis conducted.

\begin{tabular}{llll}
\hline Inputs & $\begin{array}{l}\text { Distribution Activities } \\
\text { (Technical Efficiency) }\end{array}$ & $\begin{array}{l}\text { Clinical Activities } \\
\text { (Technical Efficiency) }\end{array}$ & $\begin{array}{l}\text { Pharmacy } \\
\text { (Overall Efficiency) }\end{array}$ \\
\hline \multirow{3}{*}{ Outputs } & Doses & Clinical labor hours & Total labor hours \\
& Intravenous piggyback & No. of interventions & Total distribution output \\
& Intravenous & Soft dollar savings & Total clinical savings \\
& Chemotherapy & Hard dollar savings & Number of interventions \\
& Total parenteral nutrition prepared in-house & & \\
& Total parenteral nutrition outsourced & & \\
& Order entry without dose & & \\
& & & \\
\hline
\end{tabular}

Note: Total labor hours includes distributional, clinical and overhead labor hours 
Table 2: Description of distribution outputs used in analysis

\begin{tabular}{|c|c|c|}
\hline Output & Description & $\begin{array}{l}\text { Weighting } \\
\text { Factor } \\
\text { (minutes) }\end{array}$ \\
\hline $\begin{array}{l}\text { Medication doses } \\
\text { (Doses) }\end{array}$ & $\begin{array}{l}\text { Includes all preparation and checking, packaging and bar } \\
\text { coding of medication doses for automated dispensing } \\
\text { cabinets or medication carts. Also includes preparation of } \\
\text { kits, processing of charges, credits, and returns; and } \\
\text { medication delivery, ordering and stocking. Includes both } \\
\text { controlled and non-controlled doses, with weighting } \\
\text { adjusted to reflect controlled substances. }\end{array}$ & 3.5 \\
\hline $\begin{array}{l}\text { Intravenous Piggyback-Intermittent } \\
\text { Therapy } \\
\text { (IVPB-IT) }\end{array}$ & $\begin{array}{l}\text { Includes order review, compounding, labeling, and } \\
\text { checking of intravenous piggyback doses; along with } \\
\text { processing returns, ordering, stocking, and other support } \\
\text { functions in the sterile preparations area. Includes both } \\
\text { controlled and non-controlled doses, with weighting } \\
\text { adjusted to reflect controlled substances. }\end{array}$ & 4.1 \\
\hline $\begin{array}{l}\text { Intravenous } \\
\text { (IV) }\end{array}$ & $\begin{array}{l}\text { Includes order review, compounding, labeling, checking, } \\
\text { processing returns, ordering, stocking, and other support } \\
\text { functions in the sterile preparations area for all intravenous } \\
\text { doses. Includes both controlled and non-controlled doses, } \\
\text { with weighting adjusted to reflect controlled substances. }\end{array}$ & 4.6 \\
\hline $\begin{array}{l}\text { Chemotherapy } \\
\text { (Chemo) }\end{array}$ & $\begin{array}{l}\text { Includes order review, verification of doses and protocols, } \\
\text { order entry, compounding, labeling, double check, record } \\
\text { keeping, and special disposal or all chemotherapy doses. }\end{array}$ & 45.0 \\
\hline $\begin{array}{l}\text { Total Parenteral Nutrition-Prepared } \\
\text { (TPNprep) }\end{array}$ & $\begin{array}{l}\text { Includes order review, order entry, compounding, labeling, } \\
\text { checking, and delivery for all parenteral nutrition doses } \\
\text { manufactured in-house. }\end{array}$ & 30.0 \\
\hline $\begin{array}{l}\text { Total Parenteral Nutrition-Outsourced } \\
\text { (TPNout) }\end{array}$ & $\begin{array}{l}\text { Includes order review, order entry, checking, delivery of all } \\
\text { parenteral nutrition doses manufactured off-site. }\end{array}$ & 20.0 \\
\hline $\begin{array}{l}\text { Order entry only, not associated } \\
\text { with a dose } \\
\text { (OrdEnt) }\end{array}$ & $\begin{array}{l}\text { Includes entry of an order of any type via individual orders } \\
\text { or a protocol, including editing and discontinuing an order, } \\
\text { when no drug is actually distributed. }\end{array}$ & 1.3 \\
\hline
\end{tabular}

